\title{
The Reduction of Informal Economy Needs an Effective Tax/Fiscal System and Common Efforts of Many Factors
}

Ariet Malaj

Chairman of Approved Accountants Association

Hysen Cela

Chairman of the Institute of Authorised Expert Accountants

\section{Doi:10.5901/ajis.2015.v4n3p31}

\section{Abstract}

It's more than 25 years now that the economy of our country is part of market economy, after the decline of the communist regime. With the passing of the years this market economy is developed, perfectioned, improved and it has given signs that it can work, in fact, as a fragile capitalism, which is newly born and which is afraid to grow. As we know, we cannot take pride in a tradition that we know we do not possess. But, surprisingly and unfortunately, this economy of ours headed towards informality, which is, without exaggerating an economy accompanied with strong criminal elements. We notice that these phenomena are fought not only in the westerb world, but also in other countries, really fiercely. We can refer to China, a marker economy very industrialized, but which is still one party politically. The avoidance of taxes can be considered as a criminal phenomenon, which we are inheriting for more than 25 years now, without even speaking loudly about it, that maybe capitalism shall be born from the capital and make capital, which in fact we didn't use to have. Nevertheless, this situation of illegality and informality was ignored, which is what brought parallel social and moral damages; a situation which was allowed by the political attitude. Even nowadays, we notice that we are in stagnation, from the political point of view. Besides grave social, moral, structural and mega structural problems, this situation creates big problems even for the government to collect taxes and funds to take the role of intermediate and regulator in the economical life of the country. Accountants are the part of the financial experts, who without avoiding, can help the state to contribute in the formalization of informal economy, which to be frank cannot be considered all "black", but there are also serious private companies, which try to respect the laws and rules of fiscal "game" defined by the state, which the latter shall respect, control and make it possible for the system to function, as it was "a soccer referee".

Keywords: tax, accountant, fiscal, informal economy, law, government

\section{Introduzione}

I grandi cambiamenti nella vita politica, economica e sociale, come pure il processo inarrestabile della globalizzazione, sono stati accompagnati anche da modifiche e riforme nel sistema delle tasse. La tassazione e le tasse sono ormai un fenomeno di grandi dimensioni, che includono centinaia di migliaia di persone, che pagano le tasse, migliaia impiegati appartenenti al sistema delle tasse e migliaia di esperti e consulenti privati. A questo stato di cose " la lotta " contro l'evasione fiscale si sta intensificando sempre di piu'. Lo scopo di questa " lotta " per creare un sistema di tassazione, che cambi l'immagine della amministrazione delle tasse per essere al servizio di ogni piccolo o grande oggetto che paga le tasse, locale o straniero, attraverso la crescita delle capacita' e della qualita'. Questi cambiamenti devono rispecchiare meccanismi funzionanti, che devono avere stabilita' e sicurezza per l'imprenditoria, come pure forme e metodi stimolanti la collaborazione tra gli oggetti, che pagano le tasse e l'amministrazione pubblica. Talmente importante e' nelle condizioni della globalizzazione anche fare la distinzione tra l'evasione fiscale e la corruzione fiscale, focalizzato come un unico problema, provenienti pero' da fonti diverse. Uno appartiene ai paganti delle tasse e l'altro, a chi raccoglie le tasse, ma che sempre vanno visti come un unico problema.

Lo scopo di questo articolo e' dare delle considerazioni generali in merito ai principali principi dei sistemi della tassazione e in merito pure ai doveri e diritti basilari dell'amministrazione delle tasse e di chi paga le tasse, che pur sembrando in aspetto esterno come problemi teorici ed elementari, in sostanza sono molto importanti e stanno nelle fondamenta di ogni sistema di tassazione, dato che sono condizioni premesse per garantire il suo efficace funzionamento. 


\section{Metodologia}

Nell'articolo trattato propendendo piuttosto per il metodo dell'osservazione e meno per il metodo della classificazione e della concretizzazione ci siamo preoccupati, motivati anche dallo scopo per dare una spiegazione per l'informalita' e la sua neutralizzazione, di dare un quadro dell'indispensabilita' di un sistema di tassazione efficace. Avere la possibilita' per combinare sia l'informazione quantitativa sufficiente a causa delle posizioni direttive nell'economia, che abbiamo anche conoscitori in alti livelli delle techiche fiscali, suggerendo di aver lavorato da 35 anni contabile prima allo Stato e dopo in privato, pretenderei se mi permettessi, che l'articolo sia in verita' modestamente qualitativo e tangibile per gli studiosi. A questo proposito ci siamo preoccupati a dimostrare gli obblighi e la necessita' del miglioramento del sistema delle tasse, ma anche delle professioni contabili. E tutto questo va analizzato paragrafo dopo paragrafo. Basato nelle nostre attivita' direttive e le quotidiane pratiche valutazioni procedurali speriamo, che con questa analisi diamo un contributo positivo nella mobilizzazione e sensibilizzazione dell'opinione pubblica in generale e quella professionale in particolare per lottare ed allontanare l'informalita' e la corruzione.

\section{Perche' e' Necessario un Sistema Fiscale Efficiente?}

La compilazione e l'applicazione di un equo sistema fiscale e' importante per ogni paese, dato che tramite esso si si stabiliscono le relazioni, i diritti e i doveri reciproci nati tra le organizzazioni dell'imprenditoria da un lato e dal governo dall'altro. Dal punto di vista etico queste relazioni hanno la responsabilita' sociale di restituire alla societa' alcuni dei loro profitti, per il semplice fatto che questi profitti sono conseguenza non solo dell'utilizzo delle risorse materiali e umane, ma anche dell'infrasttutura offerente la societa'. II rispristino dei profitti per il pubblico viene realizzato attraverso il governo, che mette i piedi gli strumenti necessari dell'amministrazione e del controllo, appunto tramite il sistema fiscale e della tassazione. Tramite questi meccanismi viene garantita anche la giustizia sociale, come vengono tutelati pure i diritti dell'individuo, diritti di carattere universale.

Dunque, vanno rispettati i principi base etici e morali, la societa' garantisce la stabilita', senza la quale nessuna attivita' imprenditoriale, politica o socile non puo' essere effettuata.

Si sa che l'obiettivo principale di un sistema fiscale e' la raccolta della tassazioni e delle tasse, che servono al governo come entrate e vengono da esso usate per garantire la giustizia sociale e la tutela dell'individuo. II governo come amministratrice e osservatrice dell'attiivita' di un paese, ha l'obbligo di convertire i soldi dei contribuenti in servizi e beni pubblici per essi. II misuratore utilizzato dal contribuente e dal largo pubblico, per valutare l'equita' del sistema fiscale applicato, in ultima analisi e' la qualita' e l'utilita' dei servizi pubblici. In questo senso, la raccolta delle entrate dalle tasse e dalle tassazioni, realizzato attraverso un efficace sistema fiscale va visto in stretto collegamento con il resoconto pubblico e la responsabilita' dell'amministrazione.

Dunque che un sistema fiscale sia efficace, esso deve raggiungere il suo obiettivo principale, significante che contribuisca direttamente nella crescita e il consolidamente della fiducia del pubblico. Questo sottintende, che da un lato le autorita' fiscali (il governo) raccolga quelle somme considerate dovute e pagabili, e dall'altro lato, le organizzazzioni, le entita' imprenditoriali, ma anche gli individui, che hanno l'obbligo di pagare, pagano quelle somme richieste secondo la legislazione e le regole. II raggiungimento di questo importante obiettivo richiede, che tutte le parti, sia il governo sia i contribuenti si mettano d'accordo sulle somme, comprendano ed adempiano all'obbligo di pagare in tempo e nella giusta misura. Naturalmente, il governo nell'ambito del buon governo nell'interesse del pubblico, si richiede, che le somme raccolte le utilizzi in concomitanza col bilancio approvato, che non possono essere compilate senza tenere presente anche le aspettative del pubblico.

\section{Qual'e' L'obbligo o la Somma che deve Essere Pagata ?}

In una semplica presentazione, quello che va pagato rappresenta la somma calcolata in concomitanza con le esigenze della legislazione fiscale applicabile. Per le entita' imprenditoriali, in maniera diretta, questa somma include la tassa sul valore aggiunto all' attivita' privata, la tassa sul guadagno o anche altre tasse collegate, mentre per gli individui, essa include le tasse sugli introiti dalla paga e sulle altre entrate personali, i contributi sulle tasse sociali, sanitarie ecc. Se per la maggior parte delle piccole e medie imprese, stabilire giustamente l'obbligo non e' poi tanto difficile, questa faccenda non e' poi cosi' facile, quando bisogna calcolare l'obbligo per il resto delle imprese private, che sono piu' complesse sia per la natura dell'ambiente interno sia per il loro ambiente esterno. Oltre alla molteplicita' delle attivita' e delle operazioni questa categoria di entita' e' molto probabile, che operi anche in un ambiente economico globale, che come logica, 
supera i confini nazionali.

Sul piano nazionale e su quello internazionale attualmente si dibatte molto sulle questioni delle tasse e delle tassazioni. In modo indiretto il dibattito si estende sull'effettivita' del sistema, nella giustizia, che sottindende la pianificazione delle tasse e delle tassazioni, come pure il comportamento dei contribuenti. Sul piano internazionale, al centro di questi dibattiti sono le grandi entita', specialmente le multinazionali oppure quelle, che operano in piu' Stati, i quali attraverso una "equa pianificazione" delle tasse e delle tassazioni, che e' soprattutto legale, e' possibile, che minimizzino gli obbighi fiscali. Le multinazionali possono trasferire i loro guadagni tassabili da uno Stato a un altro in dipendenza delle norme rispettive delle tassazioni applicate in diversi Stati.

Le discussioni attuali sulle tassazioni includono sia i consulenti contabili, che i professionisti contabili, per il ruolo, che loro svolgono in questioni come l'inconciliabilita' con la legislazione fiscale, la pianificazione effettiva delle tassazioni, stabilire equamente gli obblighi, il trattamento delle richieste derivanti dagli accordi ecc.

Indipendemente dal comportamento e dall'etica di queste compagnie e dei loro consulenti sono fattori inequivocabilmente importanti per stabilire la somma, che viene chiesta e mirata ad essere pagata da esse a rispetto delle requisiti legali; anche in questi casi i governi non restano indifferenti, dato che essi non hanno un ruolo di poco conto da svolgere. Essi hanno l'obbligo e la responsabilita' per la compilazione/l'approvazione e l'applicazione delle leggi fiscali e per il rispetto degli accordi internazionali sulle tassazioni. Spetta ai governi, i quali prima di prendere iniziative sulle modifiche delle leggi e regole, valutino attentamente e tengano bene presente anche le conseguenze delle loro decisioni relative queste modifiche, allo scopo che le modifiche vengano fatte in funzione all'adempimento dei loro obiettivi prefissati. Per esempio, in caso della crescita delle norme fiscali, le compagnie multinazionali possono muovere o trasferire una parte della loro attivita' da uno Stato e questo perche' questa diventa piu' attrattiva in un altro Stato, dove le norme di interesse sono o diventano piu' attrattive.

Da quanto viene analizzato sopra, in un'economia interconnessa e globale, dove applicare un sistema fiscale effettivo non è un compito facile per i governi, i regolatori e così pure anche per le agenzie applicanti. La difficoltà si collega tra l'altro anche con gli aspetti seguenti;

- Primo, i principi sui quali si basano i codici base fiscali e la legislazione fiscale nazionale in generale, sono stati scritti e approvati molto tempo fa e sono rimasti invariati per intere decadi. Questi codici sono stati compilati molti anni fa, quando la maggior parte delle transazioni, specie di quelle di livello inernazionale erano nella forma dello scambio fisico delle merci. II fatto, che i principi e i codici fiscali sono rimasti invariati, fa sì che essi non siano indispensabilmente adatti per l'ambiente odierno delle compagnie, che hanno estensione e comportamento globale e dove la maggior parte considerevole del valore, che esse creano deriva o si genera dall'attività non materiale ( non materializzati fisicamente ).

- Secondo, i codici fiscali, ma anche la legislazione fiscale nazionale, pur essendo rivisti ed avendo la tendenza a essere modificati in continuazione, di solito sono evoluti in maniera parziale, attraverso un modello, che ha toccato solo alcuni aspetti ed ha lasciato inalterati molti altri aspetti, cosa che ha reso i codici e le leggi fiscali non integrati e non armonizzati tra essi, estremamente complessi e spesso accompagnati da molta burocrazia. Basta vedere la pratica del nostro paese dove l'approvazione di ogni anno del bilancio annuo viene preceduta da modifiche fiscali battezzate col termine "il nuovo pacchetto fiscale", il quale include modifiche nelle tasse e tassazioni in alcune leggi fiscali, in verità diventa un pacchetto "vecchio" entro un arco di tempo di un anno. Queste modifiche sono molto frequenti e non di rado, succede che esse si verifichino anche più volte entro un anno.

- Terzo, in un'economia globale, le questioni riguardanti la politica delle tasse e delle tassazioni, sebbene il governo pretenda di considerarle come questioni interne di ogni paese, non possono essere definite entro i confini nazionali, benché essi possano utilizzare legislazioni fiscali adattate, avvicinate ad un modello internazionale, essi non possono evitare le soluzioni di carattere locale. Questo, perché i governi utilizzano i loro sistemi fiscali come strumenti del loro potenziale competitivo per la regione o più largo, e in funzione allo scopo di attirare e tenere gli investimenti stranieri. Da queste operazioni, non di rado succede, che lo stimolo fiscale di un paese sia allo stesso tempo una specie di evasione fiscale da un altro paese.

A queste difficoltà vanno aggiunte anche le distinzioni esistenti tra i ritmi degli sviluppi nel rapporto finanziario per finalità generali e il rapporto per finalità semplicemente fiscali. II primo è avanzato visibilmente, tentando un utilizzo di un linguaggio comune della comunicazione, mentre il secondo sembra essere rimasto indietro. L'estensione dell'utilizzo di IFRS (International Financial Reporting Standards), oppure anche la loro totale revisione, attraverso la collaborazione tra IASB (International Accounting Standards Board) e di FASB (Financial Accaunting Standards Boord), come pure I'utilizzo e l'adozione in molti paesi di IFRS (International Financial Reporting Standards) per SME (Entities of Small and 
Medium), sono indici diretti del progresso fatto, quanto al miglioramento della credibilità del rapporto finanziario. Benché questi sviluppi abbiano la tendenza a essere utilizzati anche dal settore pubblico, il progresso in questo settore si presenta comunque piccolo ed adagio, dato che i governi in questo ambito continuano ad essere conservatori. Partendo appunto da questi sviluppi, ma anche dalle disntinzioni esistenti nei sistemi delle tasse e delle tassazioni, che usano diversi paesi, i paesi del G-20, oggi stanno discutendo della stretta e indispensabile collaborazione tra di essi, e per questo motivo hanno stabilito nelle agende di discutere, questioni legate all'iniziativa delle operazioni miglioranti per $\mathrm{i}$ sistemi delle tassazioni e delle tasse. Un tale coordinamento delle operazioni mira all'acceleramento dei ritmi dei cambiamenti e dell'adattamento dei sistemi delle tasse e delle tassazioni, allo scopo, che essi rispondano all'ambiente odierno economico globale.

Parte importante di questa collaborazione sono le iniziative, che promuovono la trasparenza dellinformazione tra le autorità fiscali. Sono pure di una rilevanza particolare anche i tentativi fiscali, fatti dall'Organizzazione per la Cooperazione e lo Sviluppo Economico (OCSE), su richiesta di G-20, che ha evidenziato sia la necessità per entrate sicure per il governo sia la necessità, per avere una stabilità fiscale.

\section{II Miglioramento del Sistema Fiscale e la Lotta Contro l'informalità}

Gli sviluppi nel miglioramento dei sistemi fiscali non possono non appartenere anche al nostro paese, il quale oggi più, che mai sembra essere orientato e deciso ad integrarsi nella famiglia naturale europea sviluppata. Sebbene le ultime due decadi si siano verificati visibili miglioramenti nel sistema delle tasse e delle tassazioni, va comunque detto, che essi sono stati insufficienti per ridurre il livello di informalità nell' economia. La scala dell'economia informale continua tuttora a restare tanto alta, che ogni operazione di miglioramento deve essere iniziata da obiettivi ancora più semplici. II migioramento del sistema va visto non separato dalla lotta frontale, che va fatta contro l'informalità. L'economia informale, la quale nella prima fase egli anni ' 90 , è servita come uno strumento di diminuizione della pressione per la alta disoccupazione ereditata principalmente dal precedente sistema, oggi dopo quasi un quarto di secolo di transizione non può continuare a rimanere in questi livelli. Benché dell'economia informale e della sua formalizzazione, si sia dibattuto tanto tra i diversi attori, quali sono le autorità governative, ed altri regolatori, inclusi anche la professione contabile (contabile e specialisti di auditing), ritengo, che sia arrivato il momento a dire, con la forza, stop a questo fenomeno.

L'esistenza e la scala dell'estensione dell'economia informale si può costatare con un'analisi abbastanza semplice. Nel nostro paese essa esiste in alcue forme, che per prima sono le attività delle imprese non registrate, queste piuttosto diffuse in aziende di piccole dimensioni e di natura di conduzione familiare; per continuare più in là nella informalità attraverso l'impegno degli assunti non registrati nelle aziende registrate, o non dichiarazione delle paghe reali per gli assunti registrati, pagando agli assunti registrati della differenza tra la paga reale e quella dichiarata all'infuori dei canali bancari, soldi in mano (in effetti è denaro informale, che viene utilizzato per evadere gli obblighi fiscali e i contributi sociali) - e viene finalizzato con l'informalità legata al rapporto finanziario incompleto, spesso, anche fraudolento, fatto dall'entità registrate, che evadono la parte dei pagamenti delle tasse e delle tassazioni, e in questa maniera, coprono una somma considerevole delle loro entrate. Si valuta, che la percentuale dell'economia informale sia in cifre molto alte.

La formalizzazione dell'economia è stata semplicemente una questione molto discussa durante questi ultimi anni dai governi, spesso accompagnata da misure di carattere educativo, restrittivo e penalizzante.

Basta citare le campagne sensibilizzanti e penalizzanti per mettere e fare usare le casse di registro, il controllo delle imprese sul terreno, le ispezioni al lavoro ecc. Comunque, i risultati sono stati esigui. II governo attuale ha fissato nella sua agenda come priorità la lotta contro l'economia informale, con lo scopo chiaro, la formalizzazione. La formalizzazione delle imprese viene vista non solo come una possibilità per accrescere le entrate nel bilancio dello Stato (entrate dalle tassse e dalle tassasazioni), ma soprattutto, come uno strumento per aumentare la competitività, diminuire la corruzione e in questa maniera, come un mezzo per creare la possibilità e pari trattamento per le imprese, cosa che si traducerebbe in più investimenti locali e stranieri.

Indipendentemente dal desiderio, o dalle azioni intraprese, sono del parere, che questa è, e resta una questione complessa e come tale richiede sforzi comuni e coinvolgimento di molti attori, come sono le autorità governative e regolatori, le autorità fiscali, le organizzazioni delle imprese e naturalmente, un ruolo importante spetta da svolgere anche alla professione contabile (contabili, i fiscalisti, e gli specialisti di auditing ecc). Finora la questione della lotta contro l'informalità risulta essere stata vista come una questione, che apparentemente sembra, che debba appartenere solo al governo, rispettivamente alle autorità delle tasse, delle tassazioni e dei regolatori, che hanno l'autorità di stabilire regole e in questa maniera, il sistema e non sembrava essere come una questione non direttamente legata alla professione contabile. 


\section{I Professionisti Contabili e il Loro Ruolo nella Lotta Contro l'informalità}

I contabili con l'esperienza tecnica e professionale, che hanno e con la loro educazione e formazione etica, giocano un ruolo chiave nell'aiuto, che danno ai clienti e ai loro assunti contribuenti, in merito agli obblighi fiscali. Un rapporto del 2007 del Forum per la Gestione Fiscale (nella cui composizione fanno parte commissionari fiscali da 45 paesi del mondo), accetta il ruolo decisivo, che hanno questi intermediari (i professionisti contabili, i fiscalisti ecc.) nel dare la certezza e garanzia del sistema fiscale, che funzioni come si deve. Dunque, che i sistemi funzionino, come si deve, si richiede il convolgimento nel giusto ruolo dei professionisti contabili, di questi "intermediari" e strumenti aiutanti, che stanno tra le imprese da una parte, e dello Stato e del pubblico, dall'altra parte.

Aiutando e consultando i datori di lavoro e i loro clienti, a comprendere gli obblighi fiscali, i professionisti contabili contribuiscono al funzionamento dei sistemi fiscali sani ed effettivi e giocano un ruolo rilevante nella stabilità economica del paese.

I professionisti contabili assicurano, che i datori di lavoro e i loro clienti comprendano le possibili opzioni fiscali previste nei codici fiscali ed aiutino ad essere competitivi nel pagamento degli obblighi fiscali.

I professionisti contabili hanno l'obbligo di rispettare i principi etici (come per esempio il Codice Internazionale dell'Etica o i codici delle organizzazioni nazionali professionali e regolatrici), e che siano guidati dai principi fondamentali dell'integrità, oggettività, e del comportmento professionale.

L'obbligo di rispettare le esigenze degli standards qualitativi, professionali ed etici, fa sì che i professionisti contabili si mostrino cauti al massimo, ed evitino il loro coinvolgimento nella preparazione delle informazioni finanziarie frodatrici 0 che disorientino gli utenti. Adempiendo a questi ruoli i membri della professione contribuiscono direttamente ed indirettamente nella lotta contro l'evasione fiscale. Comunque per far sì, che l'evasione fiscale si possa combattere non basta solo il contributo dei professionisti contabili. E' importante che il ruolo si possa armonizzare con l'utilizzo degli strumenti di stimolo (incentivi) fiscali, in maniera che quello si aspetta il governo sia adatto (soddisfi, convinca anche la parte pagante ).

Comunque, tra questi due estremi c'e' anche un'altra questione, che è complicata, perché legata all' "evasione fiscale, che arriva nel momento di pianificazione delle tassazioni adatto ", che per di più non si può considerare non legale. Questa questione presenta un dilemma difficile per i contribuenti e lo stesso anche per i professionisti contabili.

Le questioni legate al migioramento dei sistemi sono strettamene connesse con gli sforzi, che vanno fatti per la formalizzazione dell'economia. E' più, che chiaro, che questo obiettivo non si può raggiungere senza riconsiderare il ruolo importante, che devono esercitare i professionisti contabili specialmente nel funzionamento effettivo dei sistemi fiscali. Questi intermediari, che utilizzano la loro analisi per l'educazione di datori di lavoro e i loro clienti, danno consigli per le imprese, ma anche di più di questo. Comunque in fin dei conti la diretta responsabilità delle applicazioni dei sistemi fiscali resta ai contribuenti, i quali devono prendere decisioni ed azioni definitive in merito alle attività e alla compatibilità con le esigenze legali e regolatrici.

Quindi, l'utilizzo efficiente del contributo dei professionisti contabili richiede, che esso venga armonizzato il più possibile col ruolo degli altri attori. Ovviamente, ruolo di primaria importanza riveste il ruolo che devono svolgere gli Stati (i governi), che sono responsabili per la compilazione e l'applicazione delle leggi fiscali chiare, eque, ed adatte per la nostra economia. II governo deve considerare attentamente per la nostra economia e più in largo. II governo deve considerare attentamente le conseguenze delle sue decisioni per le leggi fiscali e di fare le modifiche necessarie ogni volta quando i risultati non sono quelli desiderati.

\section{Conclusioni-Raccomandazioni}

La lotta verso l'economia informale richiede l'utilizzo degli strumenti adatti e sforzi comuni non solo del governo e dei regolatori, ma anche dei contabili e specialisti di auditing, che svolgono il ruolo degli intermediari tra le imprese e le autorità statali e del pubblico in generale.

La formalizzazione dell'economia è strettamente legata al fatto di stabilire ed applicare i sistemi fiscali effettivi stabili, aventi alle loro fondamenta la giustizia nel definire gli obblighi, il trattamento indifferenziato delle imprese e che tengano presente sia le esigenze locali sia quelle internazionali.

In funzione della trasparenza, responsabilità e l'aumento della fiducia pubblica, il settore pubblico oggi si richiede, che adotti il principio dei diritti degli obblighi costatati per il rapporto finanziario. Questa adozione si rispecchia direttamente nel benessere.

E' importante valutare che i governi, i contribuenti e professionisti contabili, ciascuno ha la responsabilità 
importante e ruoli decisivi da giocare nello stabilire e far funzionare effettivamente i sistemi fiscali.

II conivolgimento della professione contabile nella lotta contro l'informalità e nella formalizzazione dell'economia, serve direttamente al miglioramento del rapporto finanziario. In questo aspetto, questo ruolo viene considerato importante per molti utenti, perché aumenta la loro fiducia e contribuisce nella stabilità economica e sociale. Oltre a questo, il bisogno per un rapporto finanziario, credibile, crescerà la competitività e di conseguenza svilupperà ulteriormente anche il mercato per i contabili e gli specialisti di auditing qualificati. Esso servirà pure come uno strumento per diminuire l'informalità anche tra gli stessi professionisti.

II settore pubblico crediamo, che deve seguire gli sviluppi nel settore privato e di adottarli per il rapporto finanziario i diritti e doveri costatati, dato che questo serve al buon governo, alla trasparenza, alla responsabilità ed accresce la fiducia pubblica.

Le continue modifiche alle leggi e regole fiscali disorientano le imprese e non contribuiscono alla formalizzazione.

\section{References}

Brooks I Dun-business \&Professional ethics for Directors, executives and Accountants, $6^{\text {th }}$ edition

IFAC Article on the development of public sector accounting

Manual for International Declarations of Quality Check, Auditing, Revision, other Securities and Services related to the editions - of IKEA, 2012

Rittenberg, Johnstone, Gramlinh-Auditing-A business Risk Approach, $7^{\text {th }}$ edition

Russell Guthrie, Executive Director, Professional Relations, and CFO,IFAC “ Governments Can't Expect to Do What Only They CanThey Must Step Ap and Cooperation on Taxation".

Taxes in Europe-2013, Published by EuraAuditlnternational, 21 st edition

The Fiscal (Tax) Legislation in the Republic of Albania ( www.tatimet.gov.al )

http://www.oecd.orgltaxl

http://www.oecd.orgltaxltransparencylabouthegloballorum.htm 